\title{
Baculovirus Surface Display Using Infuenza Neuraminidase (NA) Transmembrane Anchor
}

Irisa Trianti ${ }^{1}$, Saengchai Akeprathumchai ${ }^{1}$, Phenjun Mekvichitsaeng ${ }^{2}$, Kanokwan Poomputsa $^{1 *}$

${ }^{1}$ Biotechnology Division, School of Bioresources and Technology, King Mongkuts's University of Technology Thonburi (Bangkhuntien), Bangkok, Thailand

${ }^{2}$ Pilot Plant Development and Training Institute, King Mongkuts's University of Technology Thonburi (Bangkhuntien), Bangkok, Thailand

\section{ABSTRACT}

Baculovirus surface display has been employed as an excellent tools for presentation of foreign peptides and proteins on virus surface with native conformation, functions and immunogenicity. A baculovirus major envelope protein, gp64, or a capsid protein, vp39 are generally used as fusion partners for displaying of polypeptides on the surface of virions. Alternatively, a membrane anchoring domain of vesicular stomatitis virus G protein (VSV-G) can also be used. In this study, an influenza neuraminidase (NA) was proposed as a new membrane anchor for the display of Angiotensin II (AngII), DRVYIHPFHL, peptides. The AngII peptides were inserted into NA by replacing NA amino acid number 60-67 with AngII, and then integrated into a baculovirus genome. A recombinant baculovirus expressing the NA fusion-AngII peptides was generated from infected insect cells. Those peptides were found to express and translocated on the membrane of the baculovirus infected insect cell (Sf9 cell) as detected by immunocytochemistry using anti-AngII monoclonal antibody. Upon budding of the recombinant baculovirus progenies through the insect cells membrane, the recombinant NA-AngII peptides was acquired to envelopes of the new baculovirus progenies. The conformation of NA on baculovirus surface was not affected by the deletion, as the $55 \mathrm{kDa}$ band of NA can be detected from Western Blotting analysis by specific anti-NA monoclonal antibody. In addition, the same protein was also found by anti-AngII antibody indicating that the AngII peptides had been successfully fused with the recombinant NA. Interestingly, electron microscopy analysis demonstrated that not only the recombinant baculovirus displaying AngII peptides were generated by infected insect cells, but also the NA virus-like-particle displaying AngII peptides.

Keywords: Baculovirus surface display, Neuraminidase, AngII peptides, Virus like particles

\section{INTRODUCTION}

Baculovirus, an insect virus has been widely used as an expression system [1]. Baculovirus is able to transduce a broad range of mammalian and avian cells, therefore, it also potentially be used as gene delivery vector [2]. Interestingly, foreign protein can be manipulated to be displayed on baculovirus surface. It has been reported that baculovirus surface display is potentially be used as an efficient vaccine, such as vaccine against classical swine fewer virus [3], malaria [4] and human enterovirus 71 (EV71) [5].

The display of antigen on baculovirus surface can

\footnotetext{
${ }^{*}$ Corresponding author:

Kanokwan Poomputsa

Biotechnology Division, School of Bioresources and Technology

King Mongkuts's University of Technology Thonburi (Bangkhuntien)

49 Soi Thian Thale 25, Bang Khun Thian, Bangkok 10150, Thailand

E-mail address: kanokwan.poo@kmutt.ac.th
}

be facilitated by fusion of antigen with gp64 protein which is the major envelope protein of Baculovirus. Gp64 comprised of amino acid signal peptide domain, carboxyl proximal transmembrane domain and cytoplasmic tail domain. After gp64 expression, it will translocate to host cell membrane that control by signal peptide domain. During virus budding, the virion will take up proteins from host cell membrane to generate envelope protein, consequently, the gp64 will be incorporated in baculovirus envelope [6]. However, low number of gp64 fusion protein can be incorporated into viral membrane due to competition of fusion gp64

\section{How to cite:}

Trianti I, Akeprathumchai S, Mekvichitsaeng P, Poomputsa K

(2016) Baculovirus Surface Display using Infuenza Neuraminidase (NA) Transmembrane Anchor. J. Trop. Life. Science 6 (3): 205 -

209. 
and wild type gp64 to be uptake by virion during virus budding [7].

Thus, some other transmembrane proteins such as vesicular somatic virus glycoprotein (VSVG) has been used as an alternative. The VSVG membrane anchor approach provides higher level of display of GFP protein on baculovirus in comparison with that of gp64 fusion protein base [8]. In addition, Kolpe et al. (2012) reported that VP1 of human enterovirus 71 together with signal peptide and transmembrane domain of influenza neuraminidase (NA) can be displayed on baculovirus surface [5]. NA signal peptide and transmembrane domain fusion protein seems to have a non-polar virus distribution, unlike gp64 fusion protein that limited to the pole of baculovirus [9].

In this study, a modified full length NA fused with AngII peptide was shown to be displayed on baculovirus surface as fusion peptide. Furthermore, NA virus like particle (VLP) was also produced with AngII displayed on its surface.

\section{MATERIALS AND METHODS \\ Cells and virus}

The Spodoptera frugiperda (Sf-9) insect cells line (ATCC, USA) were grown as suspension culture in TMN-FH medium (Gibco, USA) supplemented with $10 \%$ heat inactivated fetal bovine serum (Hyclone, USA). Recombinant baculoviruses were amplified in Sf-9 cells according to Bac to Bac manual (Invitrogen, USA). The virus titter was determined by end point dilution assay [10].

\section{Generation of recombinant baculovirus displaying NA- AngII}

Recombinant baculovirus was generated according to Bac to Bac system protocol (Invitrogen, USA). A recombinant donor plasmid pFASTBac_ie1_na-angII was constructed by insertion of White Spot Syndrome Virus ie1 promoter (IE1) and na_angII into pFASTBacHT A, baculovirus transfer vector with polyhedron promoter (Invitrogen, USA). Firstly, the pFASTBacHTA polyhedrin promoter was removed and replaced with a PCR amplified ie1 promoter of WSSV. WSSV IE1 promoter was amplified by using IE1 specific primer, IE1F-5'-GTGCGTACGTAGGCTGTTTGAATCATGTTAAGG-3' and IE1 R-5'ATTAGGCGCC TATCGGACCGCTTGAGTGGAGAGAGAGA-3', pBACsurf_IE1was used as template.

AngII was inserted into influenza Neuraminidase (NA) at amino acid number 60 by overlapping PCR using pGEM-NA vector as template. After second

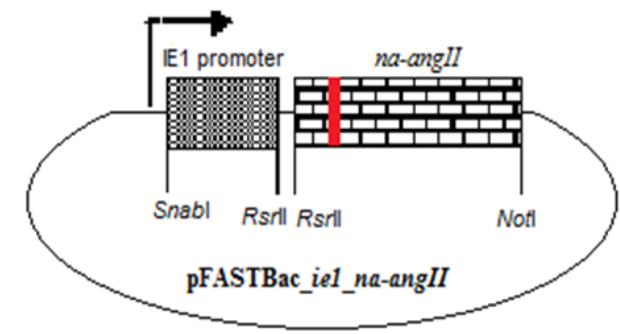

Figure 1. Recombinant donor plasmid pFastBac_ie1_naangII

round PCR, the full length NA-AngII with size of 1380 bp was generated.

The amplified PCR products were then clones into SnaBI and RsrII restriction enzyme into pFASTBac_ie1. The pFASTBac_ie1_na-angII recombinant donor plamid (figure 1) was then integrated into a baculovirus DNA (bacmid) in DH10BacTM through site-specific transposition. The positive DH10Bac E. coli colonies were selected by blue/white colony selection. The recombinant bacmid with ie1 promoter and na_angII was then extracted from white colony and was transfected into Sf- 9 cells by using cellfectin II (Invitrogen, USA) to generate recombinant baculovirus, rBV_IE1_NA-AngII.

\section{rBV_IE1_NA-AngII production}

rBV_IE1_NA-AngII was produced by infecting into Sf9 cells at multiplicity of infection (MOI) of 0.1 and harvested after 5 days post infections. The virus was collected by overlaying onto $30 \%$ sucrose and ultracentrifugation at $26,500 \mathrm{rpm}$ for 1.5 hour. The virus pellet was resuspended in PBS.

\section{NA-AngII gene expression}

To verify the expression of NA-AngII in insect cells, total RNA from infected Sf-9 cells was extracted by TRIZOL reagent (Invitrogen, USA) and cDNA was prepared according to manufacture protocol (Thermo scientific, USA). Twenty nanogram of synthesized cDNA was used as template for DNA amplification using primers specific to NA sequences and AngII.

\section{Immuno-fluorescent analysis}

Sf-9 cells were seeded in 12-well plate at cell density of $1 \times 10^{6}$ cells per well and infected with rBV_IE1_NA-AngII at MOI 0.1 for 48 hour at $27^{\circ} \mathrm{C}$. The culture medium was removed and infected cells were washed 3 times with PBS. The anti-NA and/or anti-AngII antibodies at a dilution of $1: 1000$ was used as primary antibody and a goat anti-rabbit IgG conju- 
gated with green fluorescence at dilution of $1: 5000$ were used as secondary antibody. The green fluorescence on the infected cells membrane were detected using fluorescence microscopy IX71 (Olympus, Japan)

\section{Western blot analysis}

The concentrated rBV_IE1_NA-AngII was subjected to $12 \%$ SDS-PAGE (Amersham, UK) and transferred to nitrocelullose membrane (Bio-Rad, USA). The anti-NA and/or anti-AngII monoclonal antibodies at a dilution of 1:500 was used as primary antibody and goat anti-rabbit IgG conjugated with horseradish peroxidase (HRP) at dilution of 1:5000 was used as secondary antibody. For signal detection, the protein band was developed by $3,3^{\prime}, 5,5^{\prime}$-Tetramethylbenzidine (TMB) liquid substrate (Sigma, USA).

\section{Transmission electron microscope analysis}

Carbon coated grids (EMS, USA) were floated on concentrated rBV_IE1_NA-AngII solution for $30 \mathrm{~min}$, washed with 5 drops of deionized water and negatively stained with $2 \%$ uranyl acetate solution (Sigma, USA) and examined under transmission electron microscope HT7700 (Hitachi, Japan).

\section{RESULTS AND DISCUSSION}

\section{Construction of recombinant baculovirus displaying NA-AngII}

AngII peptides, an eight amino acid peptide that responsible in controlling blood pressure in renin angiotensin system [11] was chosen as model peptides to be displayed by fusion with influenza neuraminidase (NA) and displayed on baculovirus surface. The shuttle promoter that active in both insect and mammalian cells, IE1 [12], was chosen to control NA-AngII gene expression. The NA fragment used for fusion with AngII consists of a signal peptides, a trans-membrane region, a hypervariable stalk and a globular domain to facilitate the display of AngII on baculovirus surface [13].

AngII peptide was inserted into the NA protein between amino acid residues from 60 to 67. Deletion of these amino acids at this region did not not disrupt NA conformation, in addition, the AngII peptide was expected to be located on the exposed location [14].

Figure $2 \mathrm{a}$ and $2 \mathrm{~b}$ show introduction of NA-AngII into baculovirus genome. Firstly, the recombinant transfer vector containing ie1 promoter and NA-AngII was constructed and transformed into DH10Bac E. coli containing baculovirus genome (bacmid). By site specific transposition, the NA-AngII under the control of

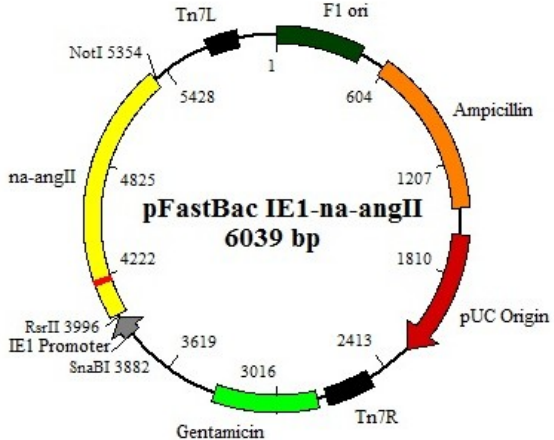

(a)

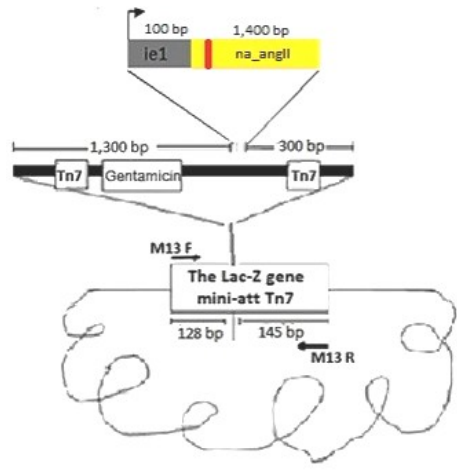

(b)

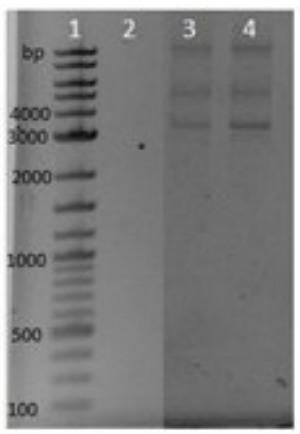

(c)
Figure 2. (a) Recombinant transfer vector, (b) Transposition of ie1-na-angII into baculovirus genome (bacmid)

(c) PCR analysis of recombinant bacmid using M13 (LacZ) specific primers. Lane 1: 2 long DNA ladder, 2: Negative control of PCR product using M13 primer and 3 and 4: PCR product of recombinant bacmid with ie1-na-angII insertion using M13 primer. (a)
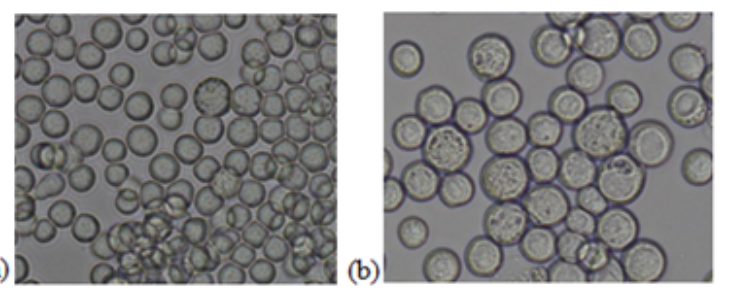

Figure 3. Sf-9 cell at 72 days in culture (a) and transfected Sf9 cells at 72 hours (b)

ie1 promoter was transferred to bacmid according to Bac to Bac system (Invitrogen, USA) at LacZ. Thus, recombinants baculoviru DH10Bac colonies become white when cultured on the LB containing X-gal (substrate to produce a blue product). The white colonies were then selected for extraction of the recombinant bacmid DNA. 


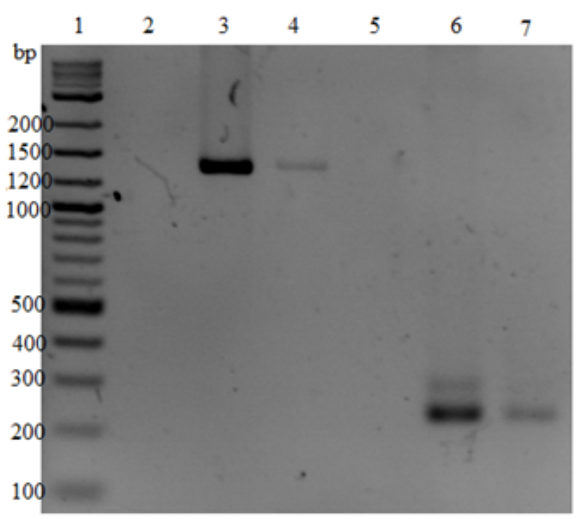

Figure 4. Reverse transcriptase PCR using NA specific primer (Lane 1-4) and NA, AngII specific primer (Lane 57) for detection of NA-AngII expression in infected cell with rBV_IE1_NA-AngII. Lane 1: 2log DNA ladder, 2 and 5: negative control without template, 3 and 6: pFASTBac_ie1_NA-AngII was used as template for positive control, 4 and 7: synthesized cDNA from infected cell was used as template

A

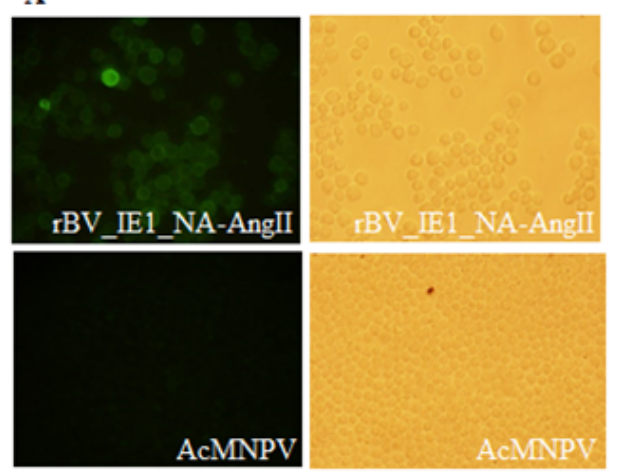

B

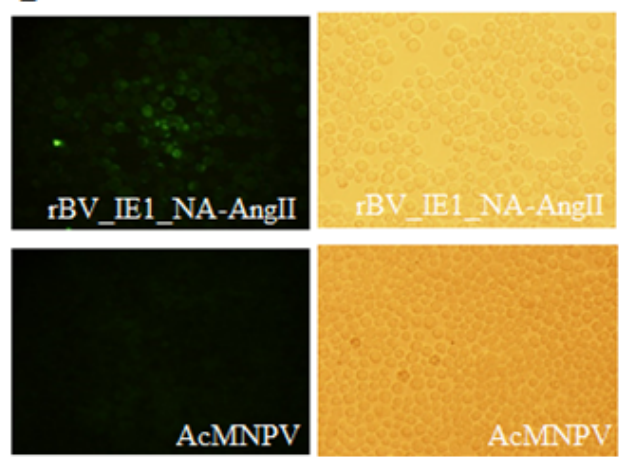

Figure 5. NA-AngII fusion protein on infected Sf-9 cell membrane. Cells were infected with wild type baculovirus, AcMNPV and recombinant baculovirus rBV_IE1_NA-AngII. 48 hours after infections, the infected cells were analyzed by immuno-flourescent using anti-NA specific antibody (A) and anti-AngII specific antibody (B), followed secondary antibody conjugated with green fluorescent.
The recombinant bacmid NA-AngII was transfected into Sf-9 insect cells using CellFECTIN II reagent (Invitrogen, USA). The recombinant baculovirus was then harvested from transfected cell culture medium when cytopathic effect of the Sf-9 insect cell was observed at 72 hour post transfection (Figure 3).

\section{Expression of NA-AngII fusion gene}

Reverse transcriptase PCR was used to verify NAAngII expression in infected Sf-9 cell with recombinant baculovirus, rBV_IE1_NA-AngII. Figure 4 shows a specific band of PCR product at $1380 \mathrm{bp}$ corresponding to the transcription of NA-AngII, when the NA specific primers were used. In addition, specific PCR product at $201 \mathrm{bp}$, which represents $\mathrm{N}$-terminal of NA (59 amino acids) with additional eight amino acids of AngII could also be detected when the NA specific forward primer and AngII specific reverse primer were used.

\section{Characterization of $r B V \_I E 1 \_N A-A n g I I$}

In order to confirm the translocation of NA_AngII to Sf-9 infected cell membrane, the immune-fluorescent analysis was performed. The Sf-9 cells were infected with rBV_IE1_NA-AngII or wild type baculovirus, AcMNPV (as control) for $48 \mathrm{~h}$. The green fluorescent were observed from infected cell with rBV-NA_AngII detected by using anti-NA antibody and anti-AngII specific monoclonal antibody, while there is no fluorescent signal can be observed from infected cells. This indicated that the NA_AngII has been expressed and translocated on the infected cell membrane, before virus budding (Figure 5).

Western blot analysis showed that NA can be detected on baculovirus surface, as the $55 \mathrm{kDa}$ band of NA can be detected by anti-NA monoclonal antibody. The same size of protein was also detected by antiAngII antibody indicating that the AngII peptides had been successfully fused with the recombinant NA and was displayed on baculovirus surface on the exposed position (Figure 6).

\section{Electron micrograph of $r B V \_I E 1 \_N A-A n g I I$}

Transmission electron microscope was performed to observe the budded virus rBV_IE1_NA-AngII that accumulated in infected cell culture medium. TEM picture shows rod shapes rBV_NA_AngII budded out from infected cell and it is expected to display NA_AngII on baculovirus surface. Interestingly, there are also NA-AngII-Virus Like Particles (VLP) can be observed in infected cell culture supernatant (Figure 7). 


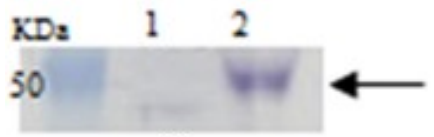

(a)

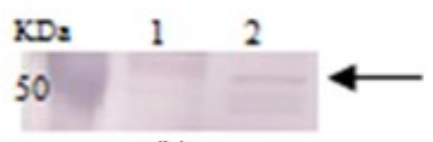

(b)

Figure 6. Western Blot analysis of recombinant BV IE1_NA-AngII against (a) anti-NA monoclonal antibody and (b) anti-AngII monoclonal antibody (Arrow indicate the NA-AngII band). Lane 1: Wild type AcMNPV, 2: rBV_IE1_NA-AngII

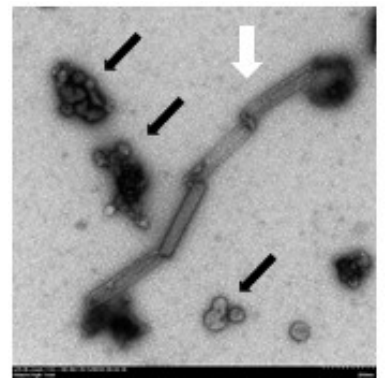

Figure 7. rBV- NA_AngII particles under transmission electron microscope. White arrow indicate rBVNA_AngII particle and black arrow indicated NA_AngII VLP

This result agree with Lai, et al (2010) that reported NA play key role in virus budding and it could facilitate the formation of NA-VLP which morphologically similar to influenza virion [15].

\section{CONCLUSION}

AngII peptides has been successfully fused with influenza neuraminidase and was located on the exposed position and the recombinant baculovirus displaying NA-AngII antigen on the surface of virus particle was successfully constructed

\section{ACKNOWLEDGMENT}

Irisa Trianti received financial support from Ministry of National Education, Republic of Indonesia through Directorate General of Higher Education (DGHE) postgraduate scholarship and technical support for research from Animal Cell Culture Laboratory, KMUTT.

\section{REFERENCES}

1. Van Oers MMV (2011) Opportunities and challenges for the baculovirus expression system. Journal of Invertebrate
Pathology 107:3-15.

2. Kataoka C, Kaname Y, Taguwa S et al (2012) Baculovirus GP-64 mediated entry into mammalian cells. J Virol 86(5): 2610-2610.

3. Xu XG, Tong DW, Chiou MT et al (2009) Baculovirus surface display of NS3 nonstructural protein of classical swine fever virus. Journal of Virological Methods 159: 259-264.

4. Yoshida S, Araki H, Yokomine T (2010) Baculovirusbased nasal drop vaccine confers complete protection against malaria by natural boosting of vaccine-induced antibodies in mice. Infection and Immunity 78(2): 595602.

5. Kolpe AB, Kiener TK, Grotenbreg GM, Kwang J (2012) Display of enterovirus 71 VP1 on baculovirus as a type II transmembrane protein elicits protective $\mathrm{B}$ and $\mathrm{T}$ cell responses in immunized mice. Virus Research 168: 64-72.

6. Rohrmann G (2013) Baculovirus molecular biology. 3rd edition. Bethesda (MD): National Library of Medicine (US).

7. Blom CO, Airenne KJ, Grabherr (2003) Technique reviews baculovirus display strategy: emerging tools for eukaryotic libraries and gene delivery. Briefing in Functional Genomics and Proteomics 2(3): 244-253.

8. Chapple SDJ, Jones IM (2002) Non-polar distribution of green fluorescent protein on the surface of Autographa californica nucleopolyhedrovirus using a heterologous membrane anchor. Journal of Biotechnology 95: 269-275.

9. Borg J, Nevsten P, Wallenberg R et al (2004) Amino terminal anchored surface display in insect cells and budded baculovirus using the amino-terminal end of neuraminidase. Journal of Biotechnology 114: 21-30

10. O'Reilly DR, Miller LK, Luckow VA (1992) Baculovirus expression vector a laboratory manual. New York: WH Freeman and Company.

11. Maurer P, Bachmann MF (2009) Immunization against angiotensins for the treatment of hypertension. Clinical Immunology 134: 89-95

12. Madhan S, Prabakaran M, Kwang J (2010) Baculovirus as vaccine vectors. Current Gene Therapy 10: 201-213.

13. Wohlbold TJ, Krammer F (2014) In the shadow of hemagglutinin: a growing interest in influenza viral neuraminidase and its role. Viruses 6: 2465-2494.

14. Castrucci MR, Bilsel P and Kawaoka Y (1992) Attenuation of influenza A virus by insertion of a foreign epitope into the neuraminidase. Journal of Virology 66(8): 4647-4653.

15. Lai JCC, Chan WWL, Kien F et al (2010) Formation of virus-like particle from human cell line exclusively expressing influenza neuraminidase. Journal of General Virology 91: 2322-2330 